\title{
Symmetry Problems in Switched Parasitic Smart Antennas
}

\author{
Elyas Palantei and David V. Thiel \\ Centre for Wireless Monitoring \& Applications (CWMA) \\ Griffith School of Engineering, Griffith University - Nathan \\ Queensland, 4111, Australia \\ E-mail: Elyas.Palantei@student.griffith.edu.au, d.thiel@griffith.edu.au
}

\begin{abstract}
Radiation pattern symmetry of a switched parasitic smart antenna is important for full $360^{\circ}$ azimuthal coverage. The ideal symmetrical beam patterns are hard to achieve in reality due to a number of crucial problems related to the need for precision design and manufacture, and the accuracy of the supporting electronics. These factors are examined on this paper.
\end{abstract}

Key words: smart antennas, switched-beam mechanism, symmetrical beam pattern.

\section{Introduction}

Switched-parasitic antennas (SPA), provide a convenient solution to multipath propagation effects (MPE) in wireless communication environments, such as fading, interference, and delay spread. The simple, symmetrical configuration of the antenna array elements, (switched active and passive), maintains directionality and impedance matching properties. Microwave, low insertion loss p.i.n. diodes are controlled by TTL voltages to switch the parasitic elements, in or out of resonance [1]. A complete SPA [2-5] was designed for plug-and-play operation with integrated control circuitry and tracking software. Various concepts of electronic control and switching techniques described previously were implemented [2].

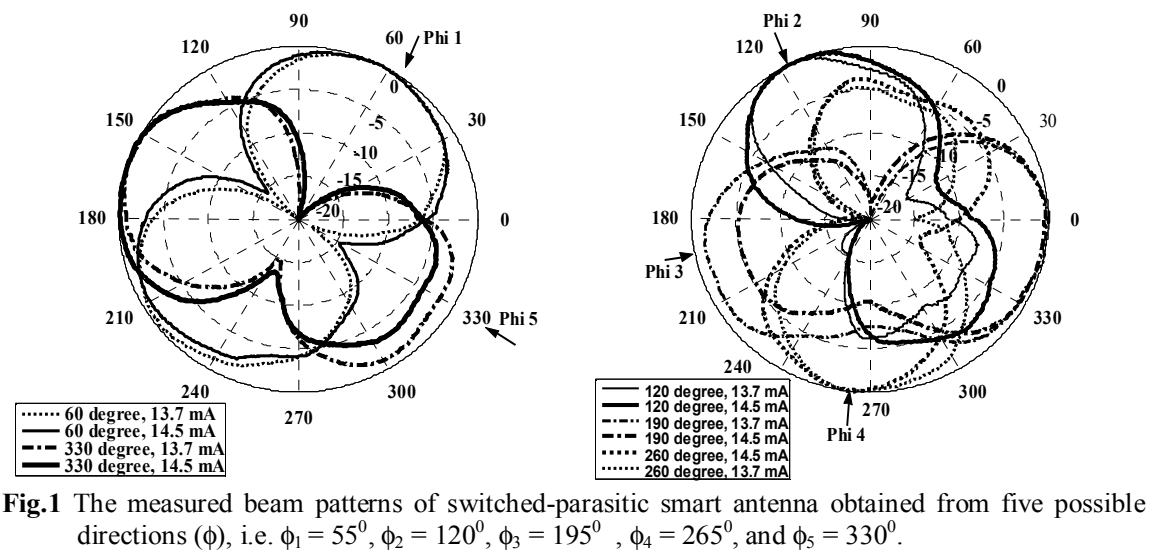

The electronics included RF signal processing and beam forming circuits mounted underneath the antenna ground plane and within the conductive skirt [6] into the physical prototype of SPA. While this smart antenna operated automatically to achieve maximum received signal levels, the beam patterns from the five possible directions were different and in some cases asymmetrical. The diode forward bias currents in each of the switching networks (SN) were adjusted and some results are illustrated in Fig.1. The skewed patterns remained and were noted for the lobe directions $\phi=195^{\circ}$ 
and $330^{\circ}$. This paper assesses other possible causes of this problem including variability in the control circuit (including microcontroller variations), RF cross coupling, DC bias effects, p.i.n. diode variation, and grounding effects.

\section{Asymmetry Investigations}

A 6 element SPA with its control circuits is shown in Fig 2. An incident RF signal is continuously received, rectified, amplified and fed to the intelligent control and beam forming circuits via the active monopole $(\mathrm{O})$. The circuit uses a predefined threshold level to categorize the RF signal as fade (poor quality, low SNR, High BER) or non fade. When a fade is detected, the antenna controller circuit is immediately set into search mode and the direction for maximum RF power is determined by switching the configuration of parasitic wires (\#A, B, C, D, and E), to open or short circuited to the ground plane. The antenna is steered through all possible directions by the bit output sequence of controller system. In the non-fade condition the antenna remains set to the current direction, however, periodic signal level surveys are undertaken routinely at predefined time intervals.

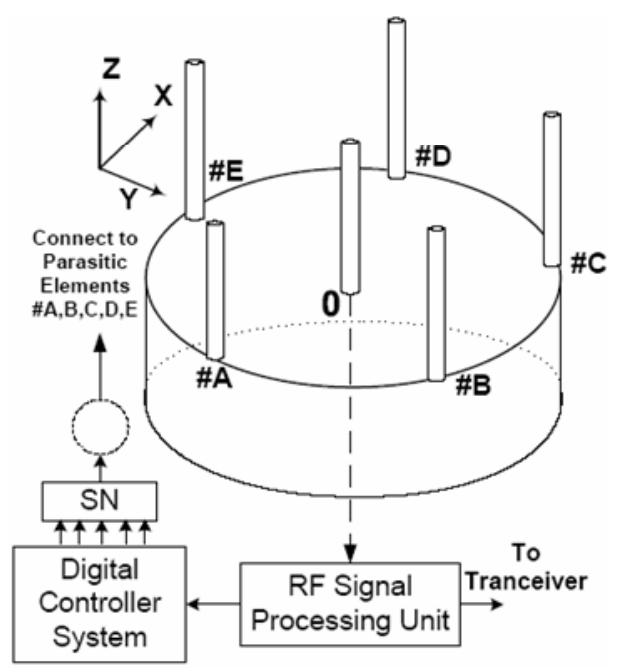

Fig.2: The circuit block diagram of switched-parasitic smart antenna

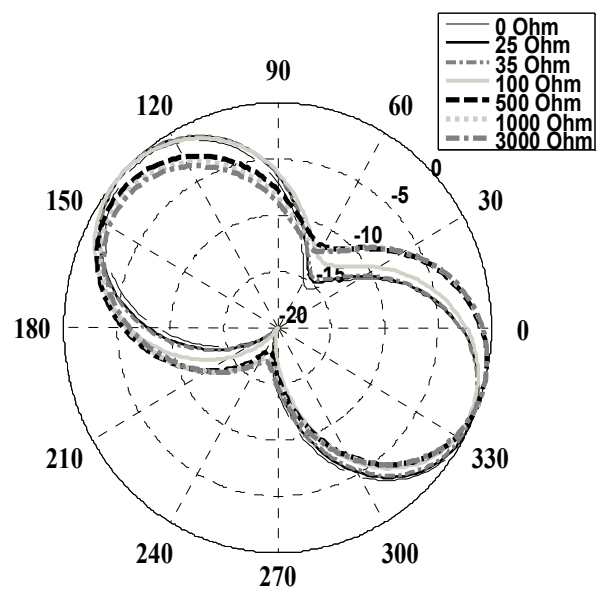

Fig.3: The computed radiation patterns resulted as the effect of both diode variation and DC bias

Uniform azimuthal coverage is possible only when the antenna provided rotationally symmetrical beam patterns separated by $72^{\circ}$. Each parasitic element has the same separation distance from the centre feed monopole. In addition, the SN components for each parasitic element are positioned exactly at its base. Experimentally however, it was observed that the set of five beam patterns are not identical or rotational symmetrical (see Fig.1). Some possible reasons for the different patterns are discussed in the next sections.

\section{a) Control Circuit Proximity Effects}

The antenna was designed with the following objectives: relatively small size, low power consumption, inexpensive construction costs, and automatic plug and play system. The control circuit consisting of the RF signal processing and beam forming circuits was created to fit into the available area below the circular ground plane. The surface mount electronics devices were soldered on a PCB layout covering this area. 
The power regulator circuit was included in this PCB to provide a constant +5 Volts DC supply to the control circuit. With one parasitic wire closely positioned to this power supply (less than $2 \mathrm{~cm}$ ) it was thought that this might provide non-uniformly distributed unwanted RF signal across the circuit board. This additive in-band noise may influence the pattern of the antenna. This is thought to be an unlikely cause.

\section{b) Diode Variations}

In the practical implementation of switched parasitic smart antenna, five microwave p.i.n. diodes (HSMP-3895 chip) were required. DC measurement [5] indicated that different diodes had different working conditions due to manufacturing variations. While all diodes displayed the same curve slope, the threshold levels were significantly different. This results in a variation in the forward resistance of each SN due to the fact that the internal resistance and capacitance of p.i.n. diode is a function of the bias current/voltage. For instance, 0.1 Volt different of bias voltage, from 0.8 to 0.9 Volts, resulted in a variation in forward resistance of about $2 \mathrm{k} \Omega \mathrm{s}$ [5]. This is can significantly affect the asymmetry of the SN total impedance at each parasitic monopole.

\section{c) DC Bias Effects}

The controller device was the one of 16F62x PIC family. One of two essential functions of the controller is to provide the DC bias to the microwave diodes. The DC bias is in the form of binary digit logic, $0(\sim 0$ Volt $)$ or $1(\sim+4.7$ Volts $)$. When measured at the applied output pin of controller device in floating condition the results appear uniform. When connected directly to the SN, variations were found to occur. These relate to the impedance load on this particular output pin as well as the current drawn from other output pins in the circuit. This problem can not be solved using a simple bias capacitor or variable resistor. The output variations generated from this device caused a $5 \%$ variation in the different DC bias voltages. The improper DC bias adjustment affects the diode impedance and so the antenna pattern including the beam shape and the beam angle direction [5]. From Fig. 1 it is evident that decreasing the bias currents to about $1 \mathrm{~mA}$, significantly changes the shape and direction of the patterns.

This effect of both diode variation and DC bias was further investigated using NEC modeling. The beam pattern of the antenna was set to the $330^{\circ}$ direction by arranging 4 parasitics \# A, B, C, and D, to be short circuited to the ground plane, and the remaining parasitic (\#E) left open circuited. The short and open circuit configurations were implemented on the NEC antenna model by inserting lumped impedance elements at the base. Short circuits were represented by setting this lumped impedance element to zero resistance. The lumped element of $10^{6} \Omega$ resistance and $30 \mathrm{pF}$ capacitance resembles a p.i.n. diode in an open circuit condition. The radiation beam produced from the antenna model was approximately symmetrical. In this test, one of the four short circuited parasitics (\#D) was grounded using a finite resistance value. Fig. 3 shows the results for a perfect short circuit, and seven different values of resistance values. The ideal short circuit of \#A,B,C monopoles, and the open circuit of \#E wire were not changed. The experimental and numerical investigations show similar behavior in that the beam shape is changed significantly. The numerical computation showed that for resistance values greater than $35 \Omega$, the pattern symmetry and direction changes noticeably. The more significant changes occurred when the resistance of $\# \mathrm{D}$ 
increased from $100 \Omega$ to $3000 \Omega$. These impedance variations are likely, given manufacturing tolerance and small variations in the forward bias voltage.

\section{d) Earthing Effects}

Inadequate local grounding of the p.i.n. diode will lead to an increase in the effective length of the parasitic monopole. This is not important when an element is set to open circuit, but can play a significant role when the element is set to short circuit. Adequate grounding and the proximity of the connections to the diode are essential requirements to the satisfactory operation of the antenna. The grounding points in the area around each parasitic element and the pad area used to solder in the p.i.n. diode was reviewed. The design of the control circuit requires strong connections between the top and the bottom sides of the circular ground plane using vias, especially at those areas immediately surrounding the base of each monopole. This will reduce the additive inductance and capacitance effects from the circuit layer of PCB. NEC modeling demonstrated that an increase in effective length of one monopole of $1.85 \mathrm{~mm}$ is sufficient to cause significant distortion of the radiation pattern.

\section{Conclusions}

Some problems that influence the symmetry of the beam patterns of switched-parasitic smart antenna have been discussed verified using NEC modeling. The manufacturing tolerance and circuit lay out are critical to antenna construction. The control circuit must be designed in such away as to minimize the coupling effects from the nearest electronics devices to the parasitic wires i.e. appropriate circuit configuration. The numerical assessment has also shown that the asymmetry impedance of the five arms of the parasitic switching network due to inappropriate DC bias set up can have a significant effect on the pattern properties.

\section{References:}

[1] D.V. Thiel, S.G. O'Keefe, and J.W. Lu, "Electronic beam steering in wire and patch antenna systems using switched parasitic elements", IEEE Antennas and Propagation Symposium, Baltimore, MD, USA., pp 534-537, 1996.

[2] D.V. Thiel and S. Smith, "Switched Parasitic Antennas for Cellular Communications," Artech House Publishers, 2002.

[3] E. Palantei and D.V. Thiel, "Electronically Switched Parasitic Smart Antenna for Wireless Communication". Conference Digest of the $9^{\text {th }}$ Australian Symposium on Antennas, Sydney, Australia, pp. 24, 16-17 Feb. 2005. On line available at http://www.ict.csiro.au/asa/asa05/

[4] E. Palantei and D.V. Thiel, "Controller Algorithms for Single Feed Switched Parasitic Antennas" on the IEEE AP-S International Symposium, USNC/URSI National Radio Science, Albuquerque, New Mexico, USA., 9 -14 July 2006.

[5] E. Palantei and D.V. Thiel, "Current Variations of Parasitic Monopoles: Its Impact on the Performance of Switched Beam Smart Antenna", Asia-Pacific Symposium on Applied Electromagnetics and Mechanics (APSAEM) 2006, University of Technology Sydney (UTS), Sydney, Australia, pp.89, 20-21 July 2006.

[6] R. Schlub, R., and D.V. Thiel, D.V., "Switched parasitic antenna on a finite ground plane with conductive sleeve," IEEE Trans. Antennas and Propagation, vol. 52 (5), pp. 1343-1347, 2004. 